Mots. Les langages du politique

$117 \mid 2018$

Les «petites phrases"

\title{
La « petite phrase » : une catégorisation méta- agissante en discours
}

"Soundbites": a meta-acting discursive categorization

Los «latiguillos»: una categorización meta-actuante al nivel discursivo

\section{Annabelle Seoane}

\section{OpenEdition}

Journals

Édition électronique

URL : https://journals.openedition.org/mots/23431

DOI : $10.4000 /$ mots.23431

ISSN : 1960-6001

Éditeur

ENS Éditions

Édition imprimée

Date de publication : 5 juillet 2018

Pagination : 91-106

ISSN : 0243-6450

Référence électronique

Annabelle Seoane, "La « petite phrase » : une catégorisation méta-agissante en discours », Mots. Les langages du politique [En ligne], 117 | 2018, mis en ligne le 05 juillet 2020, consulté le 23 avril 2022.

URL : http://journals.openedition.org/mots/23431; DOI : https://doi.org/10.4000/mots.23431 


\title{
La "petite phrase " : une catégorisation méta-agissante en discours
}

\author{
«"Les fauves sont lâchés!" : florilège des petites phrases \\ au grand destin politique» (titre du Figaro du 23/11/16)
}

Cette contribution se propose d'aborder sous un angle énonciatif et pragmatique la catégorisation de «petite phrase » dans la sphère politico-médiatique.

Des chercheurs comme Dominique Maingueneau en analyse du discours (Maingueneau, 2006, 2011 et 2012) ou Alice Krieg-Planque en sciences de la communication (Krieg-Planque, 2011), ont analysé les petites phrases comme des énoncés aphorisés : ce sont des ressorts communicationnels à forte valeur pragmatique dans une praxis journalistique qui joue un rôle majeur dans l'apparition et la propagation de ces objets. L'ancrage médiatique imprègne en effet la catégorisation de «petite phrase $»^{1}$ dès les années 1970, dans la mesure où elle «coïncide avec de vives transformations du paysage médiatique français, couplées à une forte augmentation du nombre de journalistes professionnels, ainsi qu'avec une professionnalisation affirmée des spécialistes de la communication politique» (Krieg-Planque, 2011, p. 29). Le terme est ensuite défini dans le Trésor de la langue française de 1988 comme un «propos bref d'un homme politique, qui sert à frapper l'opinion » (Brasart, 1994, p. 106) : il est utilisé par les acteurs sociaux pour décrire des fragments de discours, plus ou moins décontextualisés, qui font l'objet de reprises dans et par les médias, en raison de leur caractère remarquable ou sujet à la controverse (KriegPlanque, Ollivier-Yaniv, 2011, p. 18). Aujourd'hui, les relais du web constituent un haut lieu de propagation, de circulation et d'écho (reprises, détournements, etc.) des petites phrases, ce qui esquisse des rapprochements possibles avec le buzz (Torterat, 2010) ou les mèmes (Gautier, Siouffi, 2016).

Ces recherches se sont avant tout intéressées aux significations des petites phrases en discours et ont donc alimenté notre réflexion, mais notre éclairage se placera davantage à un niveau métadiscursif en considérant cette

1. Pour faciliter la lisibilité du propos, nous utiliserons ici les guillemets lorsque nous nous référerons à « petite phrase » en tant que syntagme catégorisant. 
catégorisation comme issue (et vectrice) d'une co-construction collective et son contexte de circulation comme producteur d'un fonctionnement interdiscursif spécifique. Notre fil conducteur est que cette catégorisation implicite autre chose que ce qui est simplement dit.

Nous en étudierons d'abord le soubassement inter- et métadiscursif : comment peut-on modéliser le jeu de (re)catégorisation et la dynamique communicative qui en émerge? L'originalité du propos tient ici dans l'effort entrepris pour aborder les liens qui unissent la propension à produire un jugement métadiscursif de saillance (qualifier un énoncé de «petite phrase», c'est d'abord en souligner le caractère saillant) et la dynamique pragma-énonciative de mise en circulation intentionnelle qu'implique la qualification de «petite phrase». Cette dynamique répond à une dialogisation ${ }^{2}$ interdiscursive agissante du point de vue communicationnel. Ce sera notre deuxième point. Enfin, l'analyse nous conduira à distinguer la «petite phrase » d'un autre phénomène de reprise : la citation. Elle s'en distingue par sa capacité à reconfigurer textuellement et discursivement l'énoncé originel, dans une énonciation modelée par une temporalité et un ancrage médiatiques. Il s'agira donc de porter l'attention moins sur le processus d'extraction d'un énoncé de son énonciation initiale que sur celui de réinjection de ce segment au cœur de l'arène médiatique avec ses rapports de pouvoir et d'opinion, ses mises en scène discursives.

Une recherche dans la base de données Europresse a dénombré 25531 documents qui font mention de «petite(s) phrase(s) », dans des contextes très variés. 5867 d'entre eux sont issus de la presse nationale généraliste française3. Nous y avons sélectionné synchroniquement une centaine d'articles, dans la période de 2015 à 2017. Ce corpus a été constitué en prenant en compte la prégnance de cette catégorisation dans le discours, soit par sa présence en titraille, soit par le développement textuel qu'elle produit. L'objectif a été d'obtenir un ensemble d'énoncés suffisamment éclairant. Il s'agissait de repérer des énoncés mettant en lumière l'articulation entre une pratique discursive et l'intentionnalité d'un énonciateur journalistique pour lequel faire mention d'une «petite phrase» devient un acte de métadiscours orienté pragmatiquement, méta-agissant.

\section{Un syntagme au creux d'une énonciation journalistique}

Il apparaît d'emblée que le syntagme « petite phrase » s'intègre lui-même régulièrement dans un dispositif de mise en saillance, et ce, quelle que soit la ligne

2. Nous entendons par dialogisme, interlocutif ou interdiscursif, le phénomène bakhtinien de coprésence de plusieurs énonciateurs, et par dialogisation le processus actif de rendre dialogique un pan de discours.

3. Àla date du 24/07/2017. 
éditoriale du journal : plus d'une occurrence sur dix se trouve dans le titre de l'article, parfois en couverture et fréquemment dans la rubrique «Actualités» ou «Événements», "Société/Polémique» (L'Humanité, 29/07/16), ou dans les pages «Décryptage» (Le Figaro, 27/02/16), «Analyse» ou «Éclairages» (Le Monde, 15/01/16), et «Débats» (La Croix, 02/03/16). Ces lieux d'apparition témoignent d'une pratique journalistique qui enclenche un processus allant de l'événement de parole à un jugement métadiscursif. Quand "petite phrase» apparaît, c'est en général pour mettre en exergue la sélection opérée par la citation, attribuée à une source parfaitement définie et axiologisée4. En désignant l'extrait retenu par l'expression «petite phrase», le locuteur journalistique en souligne les enjeux sociopolitiques et légitime ainsi son propre travail de reprise. Cette mécanique se fonde sur trois mouvements pragma-énonciatifs : 1) basculer de l'événement de parole à un jugement métadiscursif, 2) catégoriser, voire recatégoriser pour mieux montrer, 3) métaphoriser le discours.

\section{Basculer de l'événement de parole au jugement métadiscursif}

Le syntagme "petite phrase » est en fin de processus de figement et comporte donc une valeur dénominative propre : une petite phrase n'est pas une phrase qui est petite, ni même une seule phrase au sens syntaxique du terme (1) :

(1) LÂCHÉ PAR LES SIENS, PLOMBÉ PAR LES AFFAIRES, ENGLUÉ DANS LES SONDAGES [...] Dans Paris Match, Nicolas Sarkozy a toutefois lâché une petite phrase révélatrice: "Si ce n'est pas moi, Marine Le Pen peut gagner l'an prochain. Alain, qui a une image centriste, a tort de s'enfermer avec Bayrou. Dès l'instant où je ne serai plus là, les médias vont le déchiqueter.» Sarkozy l'éternel conquérant auraitil déjà acté et théorisé son futur échec à la primaire? (Marianne, 04/11/16)

L'expression "petite phrase» renvoie ici à plusieurs phrases, dont elle révèle les antagonismes politiques liés à l'actualité, laissant entrevoir un contenu implicite, laissé en filigrane : elle est «révélatrice », mais de quoi ? Ce message en suspens est ensuite explicité par le journaliste : Sarkozy aurait "acté et théorisé son futur échec à la primaire». Cette catégorisation produit deux effets : d'une part, elle crée un point d'arrêt sur le fil de l'actualité en sélectionnant certains discours signifiants pour en faire ressortir la dimension litotique5, et d'autre part, elle constitue une sorte de pivot qui fait basculer l'énonciation d'un discours de journaliste observateur à celui d'un journaliste décrypteur.

Dans cette perspective, le journaliste peut citer le segment initial appréhendé par lui comme étant une «petite phrase», comme en (1) (petite phrase in

\footnotetext{
4. C'est-à-dire placée dans un système subjectivant de valeurs et reflétant ainsi une attitude par rapport à son dire.

5. La litote consiste à dire moins pour laisser entendre plus.
} 
praesentia), ou ne pas le citer (petite phrase in absentia). Présentés en cotexte (in praesentia) ou en contexte (in absentia), les énoncés extraits et mis en circulation se rattachent à la catégorisation de «petite phrase » par des liens anaphoriques ou cataphoriques, ou des reformulations, comme dans les deux exemples suivants :

(2) Une opposition à reconstruire. Un champ de ruines... Divisée et inaudible, la droite ne s'est toujours pas remise de la cinglante défaite de Nicolas Perruchot en 2008. De son fief du Vendômois, Maurice Leroy flingue à tout-va. Certes, le député et président du conseil général (UDI) est réputé avoir la petite phrase facile et l'inimitié changeante. (L'Express, 02/09/15)

(3) Les attaques ciselées peuvent être tentantes pour se démarquer, notamment chez les challengers de Cécile Duflot. En cette fin août, tous promettent de se tenir à carreau. Jacques Boutault, maire du $2^{\mathrm{e}}$ arrondissement de Paris, veut croire qu'il y a " un consensus dans le mouvement pour tirer les enseignements de 2011. Le premier qui dégainera la petite phrase assassine aura perdu», juge-t-il. (Le Monde, 29/08/16)

\section{(Re)catégoriser pour mieux montrer}

Les paradigmes définitionnel et désignationnel soulignent la péjoration induite par la «petite phrase », "associée diversement à la surface (par opposition à ce qui serait le fond, les questions de fond, les débats de fond), [...] à la polémique [...], à l'invective, aux attaques, à la surenchère, à la mesquinerie et la bassesse [...] » (Krieg-Planque, 2011, p. 38-39). Cette péjoration se combine à l'intentionnalité d'un agir sur: sur le lecteur par la légitimation que cette (re)catégorisation pose en filigrane et plus largement sur le débat public en construction.

Les reformulations stylistiques récurrentes accentuent certaines incidences pragmatiques de «petite phrase»:

- la remarquabilité : « saillie», «jaillie», «saillie controversée», «perle»;

- la maladresse : «malentendu», «couac», «gaffe», «bourde»;

- le trait d'esprit : «bons mots», «trait d’humour»;

- l'intentionnalité agressive : «pique», «vacherie », « un coup de sang », «formule qui pique», «tacle», «escarmouches», "fulgurances vachardes», avec une série d'expansions adjectivales qui vont en ce sens, comme «assassine», "perfide», "acide», "cinglante», et des expansions prépositionnelles telles que «assertion [ou propos] à l'emporte-pièce».

Les expansions soulignent une énonciation subjectivante : les expansions adjectivales qui se rapportent à l'attribution de la source montrent des positionnements orientés axiologiquement («prononcée», «signée», "glissée», «jetée», «lâchée»), qui viennent caractériser l'émetteur de la «petite phrase ». Les relatives tendent à souligner l'impact pragmatique des propos mis en exergue : «Une petite phrase qui en dit long» (L'Humanité, 04/11/15, 
Le Figaro, 28/09/15), «La petite phrase de Macron qui ne passe pas» (Gala.fr, 02/07/17), "Brigitte Macron, la petite phrase qui fâche» (Voici.fr, 30/08/17), «la petite phrase qui touche» (Le Point, 10/11/16), «l'objectif étant la petite phrase qui tournera en boucle» (Valeurs actuelles, 01/09/16), "cette petite phrase de Wolinski qui va résonner longtemps à nos oreilles» (Libération, 04/01/16). Ces relatives, tout comme les entours prédicatifs que nous ne faisons ici qu'évoquer, mettent l'accent non seulement sur la dimension litotique des propos caractérisés comme des «petites phrases», mais aussi sur leur caractère polémique. D’autres figures rhétoriques soulignent cette dimension polémique, comme la métaphore.

\section{Métaphoriser le discours}

L'ambivalence même de l'adjectif «petite » (analysé par Krieg-Planque, 2011, $\S 4$ ) est le premier indice de la métaphorisation du discours qu'entraîne la "petite phrase». Les nombreux exemples empruntés à différents domaines de spécialité le dévoilent également : domaines météorologique (4), médical (5), culinaire (6), etc. :

(4) Dès mardi matin, à droite comme à gauche, les petites phrases ont commencé à pleuvoir ${ }^{6}$ sur les réseaux sociaux mais aussi, plus tard, à l'Assemblée nationale. (Libération, 23/03/16)

(5) Fébrilité générale sur fond de remaniement. Une recomposition du gouvernement était attendue, jeudi, avant une intervention télévisée de M. Hollande [...] La veille, c'est une autre petite phrase, prononcée par Emmanuel Macron, qui avait enflammé les esprits. (Le Monde, 12/02/2016)

(6) Valls coupe les ponts avec Hollande [...] Alors qu'il réduisait le gaz sous la casserole de l'exécutif depuis un gros mois dès qu'une petite phrase assassine fuitait, son entourage a choisi cette fois de mettre de l'huile sur le feu. (Libération, 26/11/16)

Mais l'énonciation figurale est déclenchée pour l'essentiel par la métaphore de la guerre que les journalistes de toutes les rédactions filent à l'envi. On rencontre la métaphore du «combat», de la «joute», du «duel», voire de la "guerre», avec des "armes» "affûtées», "des bombes», des «missiles», des «flingues», des "cartouches», du «gros calibre», "artillerie lourde», qui sont «dégainés» par des «snippers», ou qui «dézinguent», «dynamitent», « réduisent en ruines », comme le montrent les exemples (2), (3), (7), (8), (9), (12) :

(7) Le nouveau sniper du président [...] Il est l'auteur des piques contre la « gauche molle».

C'est Guillaume Bachelay, député quadragénaire de Seine-Maritime. Un esprit vif

6. Nous soulignons. 
qui a ciselé les pires vacheries contre François Hollande, alias «Fraise des bois» ou "Guimauve le Conquérant», avant 2012. "La présidentielle? François Hollande y pense en nous rasant " : c'est de lui. Docteur ès bons mots, roi de la petite phrase assassine, ce diplômé en philosophie est repéré par Laurent Fabius, qui en fait son conseiller. [...] Ce fan de Jacques Audiard ne s'est pas non plus gêné pour dézinguer Ségolène Royal et son mouvement Désirs d’avenir [...]. Hollande, qui savoure sa prise de guerre, enrôle le meilleur fabricant de formules du PS. (L'Express, 28/10/15)

(8) Aubry-Royal, les dessous d'une guerre froide [...] Ce 23 novembre, au 20 Heures de France 2, la petite phrase d'Aubry fait l'effet d'une bombe. Elle sous-entend que Royal s'est alliée au pacte et qu'elle ne sera pas forcément candidate. (Le Parisien, 10/12/10)

(9) Il aurait préparé sa petite phrase la veille pour la dégainer le lendemain aprèsmidi, en réaction au vote des sénateurs. (Libération, 23/03/16)

La métaphore filée de la guerre nous fait basculer dans une mise en scène de l'adversité. Elle illustre les rapports de force qui s'opèrent dans le débat public, en s'appuyant sur l'opposition attaque/défense ou contre-attaque. La tension sous-jacente mise ici en évidence se résorbe en partie par l'énonciation figurale et par la métaphore récurrente du «jeu» :

(10) [...] à continuer ce jeu de la petite phrase pleine de sous-entendus, perfide et provocatrice, vous êtes en train de vous abîmer. (Libération, 02/07/16)

Le jeu ne renvoie pas à un univers ludique mais à la manipulation (on ne joue pas avec l'autre, on se joue de l'autre), face à laquelle le journaliste s'érige en énonciateur distancié, sans doute par moments un peu cynique, en tout cas jamais dupe. Ce jeu (am)bivalent s'explique par la dynamique communicationnelle entre jugement tensif et cadre de pensée détensif, comme le montrent les exemples (11) et (12). Dans cette praxis, médiatisation et spectacularisation (Lits, 2005) se rejoignent :

(11) «Les fauves sont lâchés! » : florilège des petites phrases au grand destin politique. [...] Efficaces, spirituels, parfois agressifs, les bons mots mettent les rieurs du côté du candidat : les médias et le public adorent, tout en critiquant ce penchant pour la vacherie. Voici douze phrases ou répliques qui ont fait mouche sous la Ve République. (Le Figaro, 23/11/16)

(12) JOUTES DE POUVOIR

La politique est un sport de combat. Et, la plupart du temps, il n'est pas nécessaire de posséder un flingue pour tuer. Certains mots et des phrases bien affûtées suffisent. [...] Dès lors, la télévision veut du sang et des coups d’éclat. (Le Monde, $12 / 03 / 16)$

Si produire des figures, «c'est activer des schèmes discursifs qui modélisent certaines variations de la langue en vue d'augmenter la pertinence de 
la communication» (Bonhomme, 2005, p.41), ces métaphorisations successives concourent à co-construire en discours une relation interactionnelle entre le journaliste et son lecteur, et entre le journaliste et la petite phrase. Les schèmes énonciatifs mobilisés infléchissent l'activation interprétative du récepteur dans la mesure où « la reconnaissance de l'acte figural suppose celle de sa pertinence communicationnelle, autrement dit, elle résulte d'un rapport lisible entre le choix d'une forme particulière et du contexte où elle apparaît» (Jaubert, 2014, p.15).

En catégorisant un segment rapporté de «petite phrase», l'énonciateur aphorisant produit une tension par son activité métadiscursive, puis, en filant la métaphore, il désactive en partie cette tension pour activer plutôt la capacité interprétative et réflexive du lecteur. Cela lui permet au passage de simplifier l'apparemment complexe, ainsi trivialisé : il décrypte les attaques politiques, tout en adressant un clin d'œil complice au lectorat. Il construit alors un ethos de journaliste décrypteur, sagace et connivent. Par le biais de ces constructions figurales, ces (re)formulations constituent des jugements que portent les médias à la fois sur le propos lui-même et sur les conditions qui le font circuler. La «petite phrase» devient un levier pragmatique au cœur de la communication politico-médiatique.

\section{Jugement métadiscursif et mise en interdiscours}

Parler de «petite phrase » revient à référer à un propos de manière distanciée, en particulier dans le cas d'une aphorisation non voulue par le locuteur aphorisé. Cette distanciation pose une évaluation métadiscursive : le journaliste décrète que l'énoncé est doté d'une saillance propice à la dynamique de circulation, faisant de cette dialogisation interdiscursive un processus lui-même signifiant.

\section{Axiologiser et faire circuler}

En réactivant un propos rapporté, l'énonciateur aphorisant fait entrer le lecteur dans l'arène d'un interdiscours médiatique selon un triple paradigme :

1. une relation de connivence qui s'ancre dans une mémoire partagée, un paradigme préexistant (la représentation [l'ethos7] de l'énonciateur aphorisant, la représentation [re]construite de l'énonciateur aphorisé...) : le lecteur reconnaît les personnalités politico-médiatiques, il en a une image préalable que le discours tend à asseoir ou bien à inverser;

2. un paradigme définitionnel qui axiologise le discours : les recatégorisations, reformulations et métaphorisations successives inscrivent le discours dans une subjectivité plus ou moins marquée;

7. On entend par ethos la représentation construite en discours et par le discours. 
3. un paradigme prospectif, un à-construire avec le lecteur : cette dynamique dialogique de l'aphorisation implique une reconfiguration communicationnelle de l'énoncé originel et un acte de métadiscours agissant. Le premier énoncé, une fois repris et catégorisé comme "petite phrase», s'enrichit d'une force argumentative qui se nourrit de l'interprétation du lecteur.

Lorsqu'une petite phrase "fait le buzz», saturant le terrain médiatique sur une période déterminée, D. Maingueneau parle de «panaphorisation » (Maingueneau, 2006 et 2012). Dans le cas d'une petite phrase in praesentia, l'énonciateur (pan)aphorisant reprend à son tour des propos déjà repris par d'autres et participe ainsi à la constitution d'une chaîne médiatique interdiscursive. Ces redites ont des traits communs avec les mèmes ${ }^{8}$, «contenus auto-réplicatifs du web » (sémiotiquement mixtes : images, textes, vidéos). La diffusion rapide par les réseaux sociaux à partir des années 2000 « constitue un puissant catalyseur de la viralité : les canaux par lesquels l'information circule se multiplient » (Gautier, Siouffi, 2016, p. 11) jusqu'à la propagation virale (ou buzz, qui occupe l'attention collective). Cette viralité ne peut s'entendre qu'en termes d'unités suffisamment stabilisées. La circulation expansive a souvent pour incidence de figer (au moins en partie) ces énoncés aphorisés et commentés en «petite phrase». La catégorisation agit alors en signal de cette circulation interdiscursive, "possédant un fort pouvoir fédérateur» (Gautier, Siouffi, 2016, p.13) et mobilisateur.

La catégorisation de «petite phrase » est traitée selon un principe de résonance où chaque occurrence, commentée ou non, devient un nouveau relais. II se crée un processus d'amplification qui insiste sur la médiatisation d'un fragment, cristallisé ainsi en dérapage. La convocation d'un déjà-là, censé être partagé par le lecteur, mobilise alors une «mémoire interdiscursive» (Moirand, 2004), contribuant en retour à installer le propos cristallisé. Par chaque nouvelle occurrence, la circulation renouvelle la modalité de prise en charge de l'énonciateur aphorisant. Le contenu implicité, véhiculé à chaque réactivation, supporte des variations interprétatives en fonction des données situationnelles et contextuelles : la ligne éditoriale, le lectorat visé, l'ethos du journaliste, la date de l'article, etc. La «petite phrase » témoigne ainsi d'une capacité à s'auto-instituer en tant que vectrice d'une dialogisation interdiscursive : chaque catégorisation oriente le propos rapporté vers l'interdiscours, et les guillemets (Meteva, 2002), fréquents, en sont un bon indice. Ce basculement intentionnel vers l'interdiscours médiatique devient porteur de sens.

8. «On peut proposer de définir comme mème langagier toute nouvelle unité lexicale ou séquence syntagmatique connaissant une période de diffusion rapide accompagnée ou suivie de défigements répétés et/ou successifs, lesquels touchent tantôt au signifiant de l'expression, tantôt à son insertion discursive. Il peut donc s'agir de lexèmes (construits), comme Brexit [...], de syntagmes, comme le Grenelle de l'Environnement [...], de séquences plus longues, partiellement ou totalement figées, comme Non mais allô quoi, jusqu'aux phrases comme Vous n'avez pas le monopole de... » (Gautier, Siouffi, 2016, p. 17). 
Dans le cas d'une petite phrase in absentia, le propos aphorisé étant absent ou diffus, c'est le principe de reconnaissance de la dénomination qui prime (d'où la forte présence dans les titres) : chacun sait de quoi il retourne. Cette catégorisation revêt de fait une coloration systématiquement péjorée, car par défaut le lecteur l'associe au paradigme des énoncés placés dans une immédiateté qui les condamne à la vacuité, à la « politicaillerie » (Le Parisien, 20/04/12), « un jeu de posture [...] qui l'emporte sur l'enjeu de fond » (Le Figaro, 01/10/15), des «mauvaises habitudes» (AFP, 21/05/17) en matière de communication. $A$ cet égard, l'exercice de recueil («florilège » [11], « la phrase du jour », etc.), routinier dans la pratique journaliste, semble éloquent : il implique un processus de segmentation/sélection/compilation puis de reprise et circulation qui est orienté argumentativement vers la construction d'une représentation. Or cette représentation n'est pas construite ex nihilo, elle prend appui sur la doxa et sur des topoï (Krieg-Planque, 2011, p. 35) qu'elle consolide en retour. L'effet de morcellement du dire et d'abréviation des unités de contenu, des bribes de propos parfois rassemblés artificiellement, se trouve alors contrebalancé par ce processus unifiant de dialogisation interdiscursive.

\section{Dialogiser et mettre en scène}

Le syntagme «petite phrase» marque explicitement qu'un discours est dialogique : différents énonciateurs y interagissent. Il s’installe une altérité énonciative qui se trouve plus ou moins circonstanciée, commentée et/ou axiologisée. Ainsi, certains faits médiatiques rapportés ont pour principal enjeu de faire circuler des erreurs de communication données pour significatives de postures politiciennes (voir [5], [6], [7], [8]) ou de positionnements idéologiques jugés douteux (voir [13], infra). Dans une visée de captation et de dramatisation, ces énoncés induisent une mise en scène des productions verbales, construite bien souvent sur des postures données pour antagonistes (ce qui rend opportune la métaphore guerrière).

En cela, le processus de dialogisation interdiscursive semble décisif pour opérer la mobilisation d'un nouveau contexte référentiel et discursif. Cette opération repose sur une renégociation de l'interprétation du segment initial, car la reprise par un autre énonciateur (aphorisant) s'effectue dans un cadre discursif marqué par une prise de position (un acte de distanciation ou d'engagement) et par la nécessité de l'activation de données en dehors de l'énoncé lui-même. En qualifiant un segment aphorisé de «petite phrase », l'énonciateur aphorisant agit en discours sur le discours : cette catégorisation est méta-agissante parce qu'elle suppose une activité métadiscursive de la part de l'énonciateur aphorisant (avec une incidence sur son ethos : il se construit une image de journaliste critique, décrypteur...) ainsi qu'une activité de relecture de la part du lecteur. En même temps qu'elle la conforte, cette relecture s'appuie sur la 
représentation que le lecteur peut avoir de l'énonciateur aphorisé (comme en [13], l'association du Front national à une idéologie d'extrême droite peu démocratique correspond au dire de l'énonciateur journalistique mais également à l'orientation idéologique prêtée au lecteur du Huffington Post).

Si elles divergent par les sources énonciatives dont elles émanent, sources aphorisantes ou aphorisées, si elles divergent également par leurs fonctionnements in praesentia ou in absentia, par la présence ou non de guillemets, par les discours d'escorte plus ou moins axiologisés, il semble néanmoins que toutes les occurrences catégorisantes de "petite phrase » procèdent de ce même mouvement de mise en interdiscours, dont elles tirent justement leur force pragmatique. À chaque réactivation, on entre dans cet interdiscours médiatique qui s'imprègne de l'actualité et où se crée une relation de connivence (et un sentiment de faire communauté) par opposition au tiers aphorisé (le politique, le communicant). L'arène du discours public, avec ses rapports de pouvoir et d'opinion, ne peut alors se concevoir qu'en confrontation. Chaque emploi devient une réactivation d'un paradigme préexistant et un à-construire ensemble, qui infléchit la lecture.

La création d'un effet de figement s'articule en discours sur une saillance du fragment cité, et, du point de vue de la production comme de la réception, sur un ancrage dans la praxis journalistique. La petite phrase n'est en cela plus une simple citation. Comme événement de parole, elle repose sur un double postulat : celui d'une prise de parole identifiée à un locuteur, énoncée dans certaines conditions, et celui d'un discours fragmentable dont une partie pourrait se dissocier et se mettre à circuler dans l'interdiscours médiatique. Cette dialogisation interdiscursive provoque une déco(n)textualisation : l'énoncé originel est sorti de son contexte. Or, toute décontextualisation d'une citation originelle peut en principe générer une nouvelle pertinence 9 . Ces dispositifs se distinguent alors par leur inscription divergente dans une temporalité, un positionnement énonciatif et un ancrage médiatique.

\section{La petite phrase vs la citation : temporalité et ancrage médiatique}

La petite phrase, en tant que phénomène polyphonique coproduit par les acteurs politiques et les médias, repose sur un double soubassement : énonciatif - détachabilité et surassertion (Krieg-Planque, 2011, p. 27-29) - et communicationnel (propension à circuler). Ce double soubassement s'appuie sur

9. «La citation, parole exemplaire, court le risque de la décontextualisation. C'est le point important. Une formule sortie de son environnement discursif immédiat devient problématique; reversée alors dans un contexte plus large, elle se voit prêter une autre pertinence » (Jaubert, 2014, § 21). 
un facteur temporel qui distingue la petite phrase de la citation. La temporalité s'entend comme mouvement en cinq temps (segmenter, détextualiser, retextualiser, axiologiser et faire circuler), mais également comme temporalité dans le champ médiatique. Nous montrerons que la temporalité est un facteur sine qua non à l' «écho » d'un énoncé devenu «déclaration » puis «petite phrase».

\section{Détextualiser : segmenter et surasserter}

La citation implique une extraction et une reprise, commentée ou non par un énonciateur distinct. La petite phrase, quant à elle, se manifeste comme un énoncé «dé-textualisé» et «aphorisé» (Maingueneau, 2012). Le processus d'aphorisation induit une opération énonciative extractive, la «surassertion » qui est une «modulation de l'énonciation qui formate un fragment du texte comme candidat à une dé-textualisation [selon] une opération de mise en relief par rapport à l'environnement textuel » (Maingueneau, 2011, p.44). Cette opération repose sur la détachabilité de certains énoncés donnés pour particulièrement significatifs, avec des caractéristiques jugées prototypiques et favorables à une possible extraction-réinsertion dans d'autres (con)textes. En caractérisant des propos rapportés comme étant une "petite phrase», l'énonciateur énonce donc le fait qu’il a sciemment prélevé, décontextualisé et activé un énoncé qui implique de facto une circulation : ces aphorisations récurrentes sont configurées par l'espace médiatique et, en retour, reconfigurent cet espace.

Les modes de fonctionnement politico-médiatiques actuels sont un terreau propice à cette pratique communicationnelle coproduite et circulante (duplication des agences de presse, reprise des articles de l'AFP, etc.). La principale condition à cette circulation se fonde ainsi sur une capacité de résonance. La détachabilité ne résulte pas d'un traitement aléatoire de l'information : elle requiert concision et mémorabilité comme facteur favorable de reprise et saillance formelle ou référentielle (présence dans la titraille, chiasme, paronomase, rime ou effet de rythme, assonances et allitérations...). La deuxième condition est que le propos aphorisé implicite autre chose que ce qui est simplement dit.

\section{Retextualiser : impliciter et événementialiser du contenu}

Ces dispositifs d'aphorisation oscillent entre médiatisation et exposition médiatique. La petite phrase conçue par le communicant/le politique pour sa détachabilité se distingue de celle détachée à dessein par l'instance journalistique. L'une relève d'une aphorisation voulue, l'autre d'une aphorisation subie (Seoane, 2017). L'acteur politique peut la mobiliser en tant que forme prête à être détachée en misant sur sa capacité à créer du buzz afin de bénéficier d'une 
couverture médiatique forte à un instant donné. Le journaliste aphorisant, lui, extrait un propos d'une citation plus longue (surassertion) et lui attribue un potentiel cristallisant des enjeux politiques ou sociétaux suffisamment solide pour événementialiser (Krieg-Planque, Ollivier-Yaniv, 2011, § 9), voire polémiser, l'énoncé surasserté. Vecteur d'implicite, le segment aphorisé peut alors se charger d'une dimension litotique, un discours possible a posteriori (Torterat, 2010). Cet a posteriori latent est bien moins maîtrisable pour le politique/ le communicant, car il ouvre la voie aux métacommentaires de l'énonciateur aphorisant, le journaliste.

L'exemple (13b) illustre la manière dont le paradigme de catégorisation d'un propos cité peut opérer en discours, d'abord dé-textualisé et décontextualisé puis infléchi argumentativement. L'énonciateur aphorisé est Marine Le Pen, candidate Front national à l'élection présidentielle de 2017 , lors du débat télévisé du premier tour du 03/04/2017. Voici le passage concerné par l'aphorisation :

(13a) Cette élection porte un enjeu de civilisation, [je] constate que, après cinq ans de droite, cinq de gauche, notre pays, la France, notre pays que nous aimons tant, est livré à l'insécurité galopante, est livré au désordre économique et social, est livré au terrorisme islamiste, et livré, évidemment, à la contestation de nos valeurs fondamentales et même à la contestation de notre identité nationale. Il est aussi confronté à la mondialisation sauvage, celle qui détruit les emplois, qui délocalise les entreprises, qui dérégule à tout-va et qui aggrave la pauvreté. Je veux rendre la parole au peuple. Je veux que l'argent des Français retourne aux Français, je veux remettre la France en ordre et pour cela j'ai un projet clair, précis, je ne suis ni dans le flou, ni dans le mensonge et je voudrais dire aux Français que voter pour vraiment reprendre le pouvoir, c'est vraiment le seul vote utile.

L'énonciateur aphorisant est une ancienne avocate, Alexia Germont, engagée politiquement dans le mouvement Nous Citoyens, qui écrit un billet de blog pour le Huffington Post deux jours plus tard. L'auteur détextualise un segment final (souligné par nous) de l'intervention de M. Le Pen et en fait l'objet de son billet en le qualifiant de «petite phrase» :

(13b) LA PETITE PHRASE DE LE PEN PENDANT LE DÉBAT QUE PERSONNE N’A RELEVÉE ET QUI EN DIT LONG SUR SA VISION DE LA FRANCE

Au-delà des performances individuelles, l'organisation de ce débat doit nous inciter à tirer les enseignements qui s'imposent.

Le grand débat présidentiel ouvert aux onze candidats devait hier permettre à des millions de Françaises et de Français de mûrir leur vote. On peut douter que le but ait été atteint. Parfois confus dans la forme, très inégal sur le fond, il aura néanmoins eu le mérite de faire tomber les masques.

En effet, à l'issue de cette soirée démocratique, Marine Le Pen a laissé entrevoir son réel positionnement politique : elle a substitué à son slogan de campagne traditionnel «La France apaisée», une phrase choc, prononcée dès le début de la soirée : «Je veux remettre la France en ordre». Quasiment passée inaperçue et peu 
relevée par les chroniqueurs habituels de notre vie politique, cette phrase glaçante dans la bouche de la présidente du Front national a le mérite de la clarté. Nous aurons été prévenus, les efforts de dédiabolisation du Front national connaissent des limites évidentes [...]. (www.huffingtonpost.fr, 05/04/17)

La blogueuse caractérise le segment de «petite phrase», le reformule en "phrase choc», ajoute une série d'expansions nominales («que personne n'a relevée», «quasiment passée inaperçue et peu relevée par les chroniqueurs habituels de notre vie politique») qui donne lieu à des remarques métadiscursives («cette phrase glaçante», "a le mérite de la clarté», "faire tomber les masques») et invite enfin à une co-interprétation connivente de cet implicite par le lecteur («nous» inclusif). Les propos de l'énonciateur premier deviennent le point de départ d'un développement ultérieur, recontextualisé. La catégorisation de "petite phrase» fonctionne comme signal d'un contenu latent. Elle crée la prise de conscience d'un vide sémantique qui devient inquiétant. Pris dans ce dispositif tensif, le lecteur est amené à reconstruire ce non-dit à partir de connaissances et de valeurs supposées partagées entre lui et le journaliste, un univers de croyance donné pour commun qui permet la construction d'une représentation du tiers aphorisé : ici, cette lecture connivente passe par une implicitation des pratiques autoritaires et programmatiques du FN. Cette représentation permet d'asseoir le positionnement des énonciateurs (aphorisé et aphorisant) et d'en marquer l'opposition idéologique.

Dans cet exemple, l'altérité énonciative et idéologique s’inscrit dans une certaine temporalité médiatique, celle de l'élection présidentielle. L'énonciation d'un dit par un politique puis sa reprise critique par un énonciateur dans un blog journalistique tel que celui du Huffington Post constituent deux moments médiatiques distincts, mais tous deux portés par une même temporalité médiatique. Dans le second cas, la pratique journalistique infère une mise en information par dramatisation et se charge d'une dimension collective, alimentant le débat public; elle pose une altérité «médiacentrée» (Torterat, 2010) et métaréflexive qui «renvoie à une médiatisation (quasi-)instantanée, que les articles nomment à travers les appellatifs de "polémique" ou de "coup" [...] Ces constructions [...] favorisent moins l'information que la diffusion qui en est effectuée » (Torterat, 2010). L'exemple (13b) souligne cette événementialisation du propos aphorisé : il ne s'agit pas de le citer seulement pour le commenter mais bien d'en faire un moment-clé. La formulation de M. Le Pen n'était pourtant pas nouvelle. Elle avait déjà été utilisée par la présidente du FN dans d'autres interventions télévisées : lors de son interview par le journaliste Gilles Bouleau sur le plateau du journal télévisé de TF1, le 07/12/16 (voir [13c]), et de son intervention sur le plateau de BFM Politique, le 16/10/16, face à la journaliste Apolline de Malherbe, qui lui demandait en quoi consistait le programme de sa candidature (voir [13d]) : 
(13c) - Madame Le Pen, nous sommes à cinq mois des élections présidentielles [...] le programme de 2017 [du FN] sera-t-il peu ou prou le même [que celui de 2012], tout cela sera-t-il inchangé ou pas?

- Je veux remettre la France en ordre, en 5 ans. Je veux remettre en ordre la justice [...] Je veux remettre l'école en ordre. Je veux remettre la diplomatie en ordre. Je veux remettre l'économie en ordre, pour redonner du sens à la valeur travail. Je veux remettre en ordre l'organisation territoriale contre la désertification de nos campagnes et de nos périphéries. Je veux remettre la laïcité en ordre, qui ne cesse de reculer.

(13d) Ma candidature consistera à remettre la France en ordre. Rétablir l'ordre économique, l'ordre interne, l'ordre diplomatique [...]

Ces extraits montrent que le segment « remettre la France en ordre » tient davantage du slogan que d'un propos-événement. Sa requalification en « petite phrase », dès le titre du billet du Huffington Post, permet de justifier l'écriture d'un texte qui légitime l'énonciateur aphorisant dans sa mission d'explicitation et dans son activité métadiscursive : l'auteure prétend avoir repéré un segment signifiant aux implications graves et se pose ainsi en garante d'une démocratie en péril. Le tiers est décrié, l’énonciateur légitimé.

Le jugement métadiscursif fait ressortir un énoncé événementialisé et permet à l'énonciateur-journaliste d'impulser une dynamique dialogique interdiscursive qui lui confère un positionnement d'autorité, fondé sur un soubassement doxique déjà axiologisé. C'est en cela que la caractérisation péjorante de «petite phrase » contribue à asseoir un positionnement énonciatif au sein de l'espace médiatique.

Là où la parole du tiers se montre ostensiblement fragmentaire, sclérosée ou trompeuse, privilégiant la forme au fond, la parole journalistique, elle, se construit en filigrane comme parole qui décrypte et mobilise. En cela, le syntagme "petite phrase » a une fonction méta-agissante en discours de premier plan : elle façonne en creux un système de représentations croisées.

Cette réflexion sur la catégorisation de l'objet «petite phrase» dans un espace médiatique ouvert à différents supports et différentes formes d'énonciations nous a permis de cerner le processus de détextualisation et de réinjection d'un segment, avec ses incidences discursives : un dit initial est fragmentarisé, extrait et réinjecté dans un autre contexte énonciatif, car il est supposé condenser une posture de l'énonciateur aphorisé. Il y a alors par l'énonciateur aphorisant une mise en saillance de ce fragment qui est mis en circulation. Cette saillance implicite un dire latent. À cela s'ajoute l'opération métadiscursive de l'énonciateur aphorisant qui se positionne par rapport à ce dire aphorisé. L'énoncé détextualisé, retextualisé et catégorisé de «petite phrase » se convertit en dispositif communicationnel construit eu égard à l'ethos du locuteur originel, aux attentes supposées du public et donc aux représentations de ce même public. 
Ainsi, lorsque l'énonciateur journalistique choisit de faire circuler non une citation mais un segment qu'il dénomme « petite phrase », il fait entrer son lecteur dans le débat public en renégociant à chaque nouvelle activation du dit aphorisé les liens énonciatifs et sémantico-référentiels (qui dit, à qui il dit, dans quel contexte premier et avec quel contenu) dans la nouvelle aire discursive de la médiation de l'énonciateur aphorisant (le contenu de ce qui est dit initialement est modulé par le changement de source énonciative). Ce fonctionnement mobilise un contexte référentiel qui infléchit l'activation interprétative du récepteur : l'extraction d'un énoncé hors de son contexte d'énonciation initial ne se pose pas comme transgression par sa médiatisation, mais au contraire comme un passage éclairant. En effet, si la citation illustre un positionnement, la «petite phrase» (re)configure l'énoncé originel comme un positionnement à concevoir à l'aune de données pré-discursives (ethos préalable, représentations préconstruites, doxa) et avec une visée pragmatique particulière.

Les procédés de surassertion et de circulation permettent de faire acte de distanciation, et, par un phénomène de dialogisation interdiscursive, de mettre en scène une intentionnalité pragmatique, des figements discursifs et les conditions phénoménologiques mêmes de cette dynamique interdiscursive. Ressort communicationnel efficace, la catégorisation de «petite phrase» crée une relation de connivence ancrée dans un paradigme préexistant et un à-construire avec le lecteur. Elle fonctionne comme un levier méta-agissant au cœur d'une praxis qui lui confère la capacité de faire basculer un événement de parole en jugement métadiscursif, et en cela la "petite phrase» esquisse le passage d'un dit individuel à un dire plus collectif.

\section{Références}

Bon homme Marc, 2005, Pragmatique des figures du discours, Paris, Honoré Champion. BRASART Patrick, 1994, "Petites phrases et grands discours (Sur quelques problèmes de l'écoute du genre délibératif sous la Révolution française) », Mots. Les langages du politique, $\mathrm{n}^{0}$ 40, p.106-112.

GautIER Antoine, SIOUffI Gilles, 2016, «Introduction», Travaux de linguistique. Revue internationale de linguistique française, $n^{0} 73$, p. 7-25.

JAUBERT Anna, 2014, «Le contexte faiseur et défaiseur de figures, ou la conditionnalité de la reconnaissance figurale », dans Figures du discours et contextualisation. Actes du colloque, L. Gaudin-Bordes et G. Salvan éd., http://revel.unice.fr/symposia/figuresetcontextualisation/index.html?id=1273 (consulté le 9 mars 2018).

KRIEG-PLANQUE Alice, 2011, "Les “petites phrases" : un objet pour l'analyse des discours politiques et médiatiques», Communication \& langages, nº 168, p. $23-41$.

KRIEg-Planque Alice, Ollivier-YAniv Caroline éd., 2011, Les «petites phrases » en politique [numéro thématique], Communication \& langages, $\mathrm{n}^{0} 168$.

LITS Marc éd., 2005, Spectacularisation du politique [numéro thématique], Recherches en communication, $\mathrm{n}^{0} 24$. 
Maingueneau Dominique, 2012, Les phrases sans texte, Paris, Armand Colin.

- 2011, "Sur une petite phrase "de" Nicolas Sarkozy. Aphorisation et auctorialité », Communication \& langages, n 168 , p. 43-56.

- 2006, «Les énoncés détachés dans la presse écrite. De la surassertion à l'aphorisation », Tranel, n0 44, p.107-120.

MeteVA Elena, 2002, "La citation journalistique avec ou sans guillemets », dans Le discours rapporté, L. Rosier éd., Paris, Ophrys, p.117-124.

MoIRAND Sophie, 2004, "L'impossible clôture des corpus médiatiques. La mise au jour des observables entre contextualisation et catégorisation », Tranel, nº 40, p.71-92.

Seonne Annabelle, 2017, «Petite phrase», dans Publictionnaire. Dictionnaire encyclopédique et critique des publics, B. Fleury, M. Lecolle et J. Walter éd., Université de Lorraine, http://publictionnaire.huma-num.fr/notice/petite-phrase (consulté le 9 mars 2018).

TORTERAT Frédéric, 2010, «Quand la publicité politique se confronte au buzz journalistique : le cas des dérapages verbaux traités dans une rubrique de quotidien », Signes, Discours et Sociétés, no 5 , http://www.felsemiotica.org/site/wp-content/uploads/2014/10/ Signes-discours-et-soci\%C3\%Agt\%C3\%Ags-5-Communication-et-discours-politiquesactualit\% $3 \%$ A9s-et-perspectives.pdf (consulté le 9 mars 2018).

\section{Résumé / Abstract / Compendio}

\section{La «petite phrase» : une catégorisation méta-agissante en discours}

Cette contribution se propose d'aborder sous un angle pragma-énonciatif la catégorisation de «petite phrase», appréhendée comme ressort communicationnel. L'ancrage politico-médiatique y sera articulé à la problématique d'une mise en circulation et d'une intentionnalité de l'énonciateur journalistique. Faire mention d'une "petite phrase» devient alors un acte de métadiscours orienté pragmatiquement et donc méta-agissant.

Mots-clés : petite phrase, catégorisation, presse, énonciation, aphorisation

\section{"Soundbites": a meta-acting discursive categorization}

This contribution proposes to study from a pragma-enunciative point of view the categorization of "soundbites", understood as communicative nuts and bolts. Their political and media anchoring will be articulated with the themes of publicizing and of the intentionality of the journalistic enunciator. Mentioning a "soundbite" becomes a metadiscursive act that is pragmatically oriented and thus a meta-acting process.

Keywords: soundbite, categorization, press media, enunciation, aphorisation

\section{Los «latiguillos»: una categorización meta-actuante al nivel discursivo}

Se trata de estudiar aquí la categorización de «latiguillo» (una declaración polémica) desde un punto de vista pragmático y enunciativo. Su dinámica comunicativa se apoya en el espacio mediático y se articula con el tema de la circulación de la intencionalidad del enunciador periodístico. Mencionar un «latiguillo» se convierte en un acto meta-discursivo orientado pragmáticamente.

Palabras claves: frase corta, categorización, prensa, enunciación, aforismo 